\title{
Ultrasonic Intensification of Mass Transfer in Organic Acid Extraction
}

\author{
Oleg M. Gradov *, Yulia A. Zakhodyaeva, Inna V. Zinov'eva (D) and Andrey A. Voshkin (D)
}

check for

updates

Citation: Gradov, O.M.; Zakhodyaeva, Y.A.; Zinov'eva, I.V.; Voshkin, A.A. Ultrasonic Intensification of Mass Transfer in Organic Acid Extraction. Processes 2021, 9, 15. https://dx.doi.org/ 10.3390/ijms22010051

Received: 24 November 2020 Accepted: 21 December 2020 Published: 23 December 2020

Publisher's Note: MDPI stays neutral with regard to jurisdictional claims in published maps and institutional affiliations.

Copyright: () 2020 by the authors. Licensee MDPI, Basel, Switzerland. This article is an open access article distributed under the terms and conditions of the Creative Commons Attribution (CC BY) license (https: / creativecommons.org/ licenses/by/4.0/).
Kurnakov Institute of General and Inorganic Chemistry, Russian Academy of Sciences, 31 Leninsky Prospect, 119991 Moscow, Russia; yz@igic.ras.ru (Y.A.Z.); iz@igic.ras.ru (I.V.Z.); voshkin@igic.ras.ru (A.A.V.)

* Correspondence: lutt.plm@igic.ras.ru

\begin{abstract}
The mechanism of ultrasonic extraction was discovered and analyzed in detail for the liquid membrane technique from the consideration of the specific features of the radial vibrations of a droplet of the dispersed phase placed into an immiscible continuous phase subjected to ultrasonic irradiation. Analytical formulas were derived for the rate of mass transfer as a function of the amplitude of acoustic pressure oscillations and the time of ultrasonic treatment of an extraction system. Conditions for achieving the maximum efficiency of the extraction of a substance under the stimulating effect of ultrasound were analyzed. A nonlinear equation was derived for the radial vibrations of a spherical droplet of the dispersed phase in an immiscible continuous phase under forcing in the form of acoustic pressure periodically changing with time. Experimental study of the dependence of sulfosalicylic acid distribution on time in an aqueous two-phase system with ultrasound shows good agreement of experimental results with the calculations performed.
\end{abstract}

Keywords: pressure drop; acoustics; ultrasonic irradiation; nonlinearity; resonance frequency; ecofriendly extraction; interphase distribution; aqueous two-phase system; poly (ethylene)glycol; intensification

\section{Introduction}

Through the ages, people successfully used various extraction methods in one or another form to improve their wellbeing by increasing the quality of the implementation of the developing capabilities of chemistry and physics throughout the entire period of human civilized existence [1]. In particular, the problems of the recovery of metals from different substances [2-6] and the extraction of other chemical compounds from liquids [7-15] are constantly enriched with new solutions, the corresponding schemes of practical implementation, and the possibilities of using the correlating achievements from related branches of science. This includes the use of ultrasound, which can have a substantial effect on the results of various experiments from the purification of soil from petroleum products [16] to metal hardening [17]. The ability of ultrasound to enhance various physicochemical processes is based on its capability of altering particular characteristics of the phenomenon under consideration and, consequently, affecting the final product in a controlled process. In particular, a methodology for ultrasonic soil purification from inorganic contaminants was developed in [18], and the dependence of sonochemical reactions on the ultrasound frequency with allowance for the specific features of the cavitation process was studied in [19]. Among the latest achievements is also the successful use of ultrasound to enhance the removal of various toxic organic dyes and heavy metals using nanoparticles loaded on activated carbon [20]. Ultrasound is often used to extract various biologically active substances [21], polysaccharides from plant materials [22], pesticides from soil [23], and rare earth elements from rocks [24]. Ultrasonic extraction proved to be an environmentally safe method for the recovery of substances [25]. Its advantages over other extraction methods are a decrease in the extraction time and energy consumption and an increase in the yield of products and the preservation of the biological activity of recovered biomolecules due to 
lower extraction temperatures [26]. In recent years, ultrasonic liquid-liquid extraction of organic acids from aqueous solutions is of greatest interest [27]. Optimal conditions for the use of ultrasound for implementing continuous solvent extraction of lactic acid from fermentation broths were determined by sequential sampling [28]. The study [29] in which conditions for the effective use of ultrasonic intensification were experimentally found for the recovery and separation of antioxidants from wheat chaff in an aqueous two-phase system can also be cited as an example that confirms the existence of a large variety of objects for the use of ultrasonic extraction. Although conditions close to optimal ones can be found in a number of cases experimentally using a simple examination of possible values of experimental parameters, nevertheless, the determination of such conditions based on revealing and studying mechanisms that constitute the physicochemical essence of the processes under study is the most preferable, since, among other things, it allows one to construct the configuration and scheme of the most effective implementation of the assigned task. For example, it is possible to affect extraction processes in the necessary direction by creating conditions for the implementation of the mechanisms of nonlinear acoustics, as was shown in [30-33]. Even in the simplest case of ordinary irradiation of the working volume of an extractor with low-power ultrasound, certain features caused by external action can be revealed that can substantially change the efficiency of the process. In this study, such an important characteristic of extraction as mass transfer implemented under the conditions of a breakup of the original solution containing the target component (the compound being extracted) into droplets is investigated for the case where external ultrasonic radiation directly affects this process by forcing the droplets of the original solution to perform radial vibrations, thus substantially changing the pattern of the recovery of the extract in comparison with the conventional scheme for the treatment of a liquid. In this case, the used ultrasound intensities are significantly lower than the thresholds required to excite such nonlinear effects, for example, cavitation, which can radically change the essence of the processes under consideration. Based on the derived nonlinear equation for the radius of a droplet, the parameters of forced oscillations of the spherical surface in the presence of ultrasound have been studied, and the specific features of mass transfer through the oscillating phase boundary were analyzed. The revealed specificity of the dependence of the extractant concentration on the time and intensity of ultrasonic irradiation can be a determining point in choosing the efficient regime of the use of ultrasonic extraction.

\section{Theoretical Part}

To perform the well-founded evaluation of the effect of ultrasound on mass transfer under the conditions of the breakup of the dispersed phase (for example, the original solution containing the target component), it is necessary to consider the problem of the pulsations of a droplet that is in an immiscible continuous phase (a solution of the extractant) in which pressure changes with time $t$ under the effect of ultrasound. As a result of solving this problem, it is possible to determine the character of the oscillations of droplet radius $R(t)$ to calculate the variation in the droplet surface area on which mass transfer takes place. Although lots of drops are present in the extractant, the interaction between them weakly affects the final extraction result, so those basic specifications of ultrasonic recovery of target compound completely connected with processes that are occurring inside of every single drop, i.e., the quantitative results of the whole procedure are defined by the simple summary of appropriate indicator for every drop, in which, presumably, identical reactions have proceeded. It should be noted that a similar approach is justifiably applied in the cavitation investigation [34].

\subsection{Problem Formulation and Original Relationships}

If $\rho_{1}(r, t)$ is the density of the droplet and $\rho_{2}(r, t)$ is the density of the surrounding liquid, their common equation of motion that describes the variation of the radial velocity $u(r, t)$ 
and pressure $p(r, t)$ can be written in the spherical coordinate system, the origin of which $r=0$ is placed in the center of the droplet, as follows [32]:

$$
\frac{\partial u}{\partial t}+u \frac{\partial u}{\partial r}=-\frac{1}{\rho} \frac{\partial p}{\partial r}
$$

It is important to note that considering the spatial nonuniformity of the acoustic pressure defined by the ultrasound wavelength is not significant because of the large difference in size between this length and the radius of the drop. Therefore, the case is considered when a drop is subjected to a comprehensive homogeneous compression (or stretching) by the external pressure of a surrounding medium. The compressibility of the liquid due to its small contribution to the final result can be neglected; therefore, the continuity equation has the following simple form:

$$
\frac{\partial}{\partial t}\left(r^{2} \rho u\right)=0
$$

The solution of Equation (2) is related to the velocity of the droplet surface $U(t)=d R / d t$ by the formula

$$
u(r, t)=\frac{R^{2}(t) U(t)}{r^{2}}, r \geq R(t)
$$

Let the droplet in the initial state be in equilibrium with the surrounding medium and has the radius $R_{0}$ and pressure $P_{10}$. In that case, from the Tait equation of state for the condensed media [34,35], the following relationship can be written at an arbitrary time point:

$$
P_{1}(t)=A\left\{\left(\frac{R_{0}}{R(t)}\right)^{3 \gamma}-1\right\}+P_{0}+\frac{2 \alpha}{R_{0}}, P_{10}=P_{0}+\frac{2 \alpha}{R_{0}}
$$

where, $A$ and $\gamma$ are constants which are determined empirically, $\alpha$ is the surface tension, therefore $2 \alpha / R(t)$ determines the pressure caused by it, and $P_{0}$ is the hydrostatic pressure of the surrounding liquid in the initial equilibrium state. The equality of pressures at the droplet boundary leads to the relationship:

$$
A\left\{\left(\frac{R_{0}}{R(t)}\right)^{3 \gamma}-1\right\}+P_{10}=P(R)+\frac{2 \alpha}{R(t)}
$$

From Equation (5), we can write the following relationship for the pressure $P(R)$ on the droplet surface:

$$
P(R)=A\left\{\left(\frac{R_{0}}{R(t)}\right)^{3 \gamma}-1\right\}-\frac{2 \alpha}{R(t)}+P_{0}+\frac{2 \alpha}{R_{0}}
$$

Since in the equilibrium state $p(r \rightarrow \infty)=P_{\infty}$, in the case where a cavity is subjected to the pressure of ultrasonic irradiation $P_{m} \sin \omega t$ with the amplitude $P_{m}$ and frequency $\omega$, the pressure at infinity can be written as

$$
P_{\infty}=P_{0}+P_{m} \sin \omega t
$$

By introducing the velocity potential $\varphi(r, t)(u=\nabla \varphi)$, Equation (1) can be integrated concerning the radius $r$ from $r$ to $\infty$; as a result, we have the relationship:

$$
\frac{\partial \varphi}{\partial t}+\frac{u^{2}}{2}+\frac{P_{\infty}-p}{\rho}=0
$$

since for $r \rightarrow \infty$ there are the equalities $\varphi=0, u=0$, and $p(r \rightarrow \infty)=P_{\infty}$ and the density is related to the pressure by the equation of state. As it follows from Equation (3) that 
$\varphi=-U R^{2} / r$, the following equation is derived from Equation (8) on the droplet surface with allowance for Equation (5) and Equation (7):

$$
R(t) \frac{d^{2} R}{d t^{2}}+\frac{3}{2}\left(\frac{d R}{d t}\right)^{2}+\frac{1}{\rho_{2}}\left\{P_{m} \sin \omega t+2 \alpha\left[\frac{1}{R(t)}-\frac{1}{R_{0}}\right]+A\left[1-\left(\frac{R_{0}}{R(t)}\right)^{3 \gamma}\right]\right\}=0
$$

Using Equation (9), it is possible to solve any problems of the nonlinear time behavior of the spherical surface of a droplet from complex deformation to simple pulsations under the effect of ultrasound. An analysis of the characteristics of mass transfer intensification in an extractor under the effect of ultrasound relies considerably on the quantitative indicators of pulsations calculated using Equation (9). It should also be noted that Equation (9) is similar in its main features to the equation describing the behavior of a gas bubble in a liquid [34] under the effect of ultrasound. It should be noted that, because of the great difference between the drop size and the ultrasound length, acoustic pressure is spatially homogenous at the radius order length $R_{0}$ and only oscillates in time with set frequency $\omega$.

\subsection{Calculation of the Intensity of Mass Transfer under the Effect of Ultrasonic Vibrations for a} Single Droplet of the Dispersed Phase

In the linear approximation, the radius of a droplet of the dispersed phase located in an immiscible continuous phase subjected to ultrasonic irradiation can be represented as

$$
R=R_{0}+\delta R_{m} \sin \omega t+\delta R_{m 2} \sin 2 \omega t+\ldots
$$

Of greatest interest is the case where the effect of ultrasound on mass transfer processes shows itself at relatively low intensities of its use, which excludes the development of undesirable by-effects of force character (heating, cavitation, and so forth), i.e., when $R_{0} \gg>R_{m}$. It should be noted that ultrasound can have a significant effect on the formation of droplets used in extraction. A detailed study of this process was carried out in the following works [30-33]. It is not noticeably important for subsequent calculations since even in the most general cases it changes only the magnitude of an equilibrium radius $R_{0}$. However, for considered small values of ultrasound intensities, those changes are inessential.

Consideration of the effect of an acoustic signal on mass transfer processes in a droplet should begin with a detailed analysis of the main features of the extraction process. If a droplet contains $N$ particles of the compound being extracted with the characteristic size $L$ (it can also be foreign molecules), the main parameters of the extraction process can easily be expressed using formulas convenient for practical application when certain natural conditions are satisfied. First, the distribution of particles over the volume of the droplet should be uniform, and transient processes for its attainment after the recovery of the next portion of the compound being extracted should occur for the time interval smaller than the period of ultrasonic vibrations. Second, all particles in the near-surface layer of the droplet with the thickness $L$ are assumed to be instantaneously extracted; therefore, the next moment of extraction will take place after the time interval $\Delta t$, for which a new portion of extracted particles moving with the velocity $w_{d}$ can fill the same layer of the droplet that is $\Delta t=L / w_{d}$. It should be noted that, for the motionless radius of a droplet (the absence of ultrasound), the time interval $\Delta t$ for the filling of the near-surface layer with particles is determined by the rate of diffusion processes or by an acceleration of the droplet and so on; therefore, the present scheme for the description of extraction dynamics is also applicable in the case where ultrasound is absent. Figure 1 explains the interrelationship between the duration of the extraction cycle $\Delta t$ and the depth $L$ of the zone of particle recovery. 


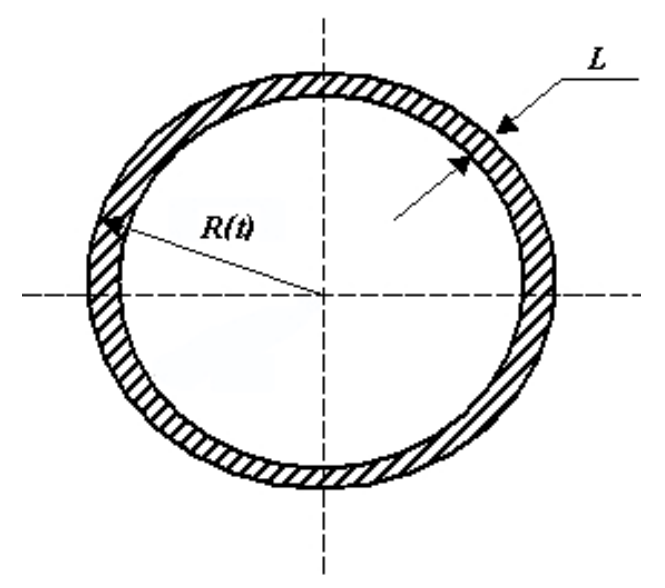

Figure 1. Schematic representation of a model for cyclic extraction of particles with the characteristic size $L$ from a droplet of the liquid with the variation in its radius $R(t)$ under the effect of ultrasound.

Thus, the introduction of the simplified calculation discrete in time extraction scheme based on the assumption that processes of the internal motion in the drop (ultrasonic flows or diffusion transfer) supply of the required recover particles to the drop surface. The truth of this assumption should be verified by the reconciliation of theoretical calculations to experimental data.

The mentioned approach sometimes proves to be more productive in comparison to the implementation of strict mathematical pieces of evidence, which cannot always be carried out without numerous new assumptions.

Let $d n$ be the number of extraction cycles for the time interval of $d \tau=\Delta t d n$. In this case, the number of particles $d N$ extracted from the droplet is determined by the formula:

$$
d N=-4 \pi R^{2} L n_{0} d n \equiv-4 \pi R^{2} L \frac{n_{0}}{\Delta t} d \tau, n_{0}=\frac{3 N}{4 \pi R^{3}}
$$

Here, $n_{0}$ is the approximate value of the density of the particles being extracted in the droplet.

The integration of Equation (11) after inserting their $\Delta t$ yields the following relationship for the time variation of the number of particles in the droplet:

$$
N(\tau) \cong N_{0} \exp \left\{-3 w_{d} \tau / R\right\}
$$

It can be seen from Equation (12) that the number of particles exponentially rapidly decreases from the initial value $N_{0}$ with time, the characteristic period of which depends on the velocity of microstreams inside the droplet. This number will depend on the amplitude and frequency of ultrasound in the case when corresponding oscillations of the radius $R(t)$ cause the most intensive motion of the liquid inside the droplet with the velocity:

$$
w_{d}=\frac{d R}{d t} \cong \omega \delta R_{m} \sin \omega t
$$

Taking into account diffusion and other processes, which can exert influence on the velocity of microstreams inside the drop, complicates essentially the expression of Equation (13) for $w_{d}$. However, in special conditions of ultrasound domination over them this expression is valid, and its use simplifies calculations significantly. The detail analysis leads to the final formula

$$
N(\tau) \cong N_{0} \exp \left\{-6 \delta R_{m} \omega \tau /\left(\pi R_{0}\right)\right\}
$$


The form of the dependence on the amplitude and frequency of ultrasound can be determined because of solving Equation (9), which can be described using Equation (10) by the formula:

$$
\frac{\delta R_{m}}{R_{0}} \frac{\omega^{2}-\Omega^{2}}{\Omega^{2}}=\left(\frac{\delta R_{m}}{R_{0}}\right)^{3}\left\{\Gamma_{1} \Gamma_{2}-\Gamma\right\}+P_{a}
$$

In Equation (14), which is the essentially nonlinear equation related to the variable $\delta R_{m} / R_{0}$, the quantity $P_{a}$ that determines the amplitude of acoustic pressure in the dimensionless form is introduced and the symbol $\Omega$ is used for the frequency of the natural oscillations of a droplet according to the formulas:

$$
\begin{gathered}
P_{a}=\frac{P_{m}}{2 \rho_{2} R_{0}^{2} \Omega^{2}}, \Omega^{2}=\left(3 \gamma A-\frac{2 \alpha}{R_{0}}\right) \frac{1}{\rho_{2} R_{0}^{2}}, \Omega_{1}^{2}=\frac{3 \gamma A}{\rho_{2} R_{0}^{2}} \\
\Gamma=\frac{(3 \gamma+1)(3 \gamma+2) \Omega_{1}^{2}}{6} ; \Gamma_{1}=\frac{2 \omega^{2}-\Omega^{2}-3 \gamma^{2} \Omega_{1}^{2}}{\Omega^{2}} ; \Gamma_{2}=\frac{5 \omega^{2}-2 \Omega^{2}-\Omega_{1}^{2}(3 \gamma-1)}{2\left(4 \omega^{2}-\Omega^{2}\right)}
\end{gathered}
$$

For parameters in Equation (14), we have the following definitions:

The exact solution to cubic Equation (15) can be written using Cardano's formula, but it is rather bulky, and its analysis is not simpler than the study of the original Equation (15). General representation of the behavior of the vibration amplitude of the droplet surface with a change in the frequency and amplitude of acoustic pressure can be obtained from Figure 2, which shows the dependence of the dimensionless amplitude $A_{0}(\omega)=\delta R_{\mathrm{m}} / \delta R_{\max }$ $\left(\delta R_{\max }\right.$ is the maximum value of amplitude for the chosen value of $\left.P_{a}\right)$ on the pump frequency $\omega$ divided by the natural frequency $\Omega(z=\omega / \Omega)$.

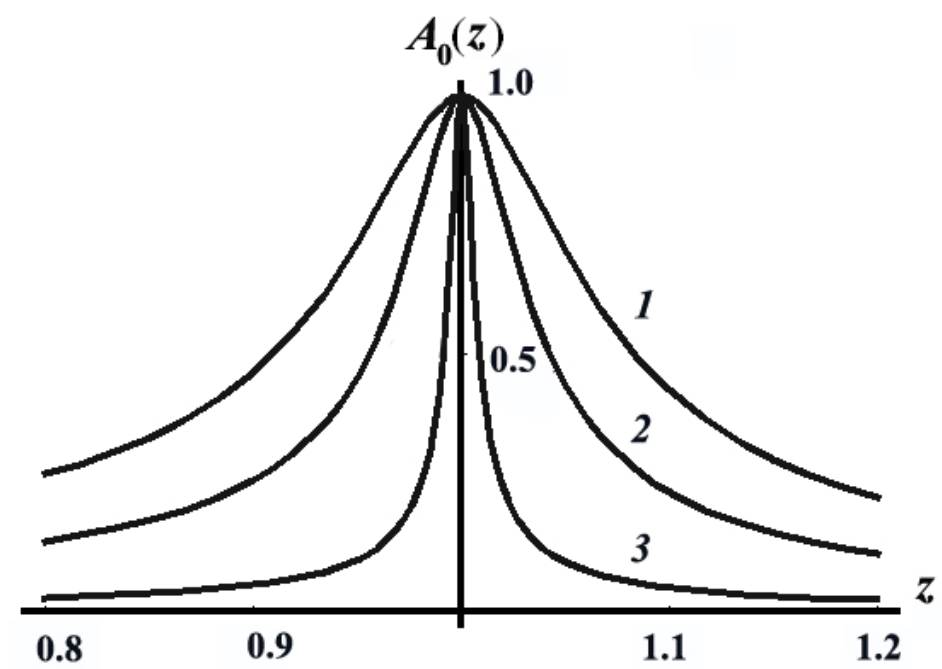

Figure 2. Dependence of the dimensionless vibration amplitude of the droplet surface $A_{0}(z)=\delta R_{m} / \delta R_{\max }$ on the pump frequency $\omega$ divided by the natural frequency $\Omega($ where $z=\omega / \Omega$ ) for some values of the dimensional less amplitude $P_{a}$ of acoustic pressure: $1-P_{a}=0.1 ; 2-P_{a}=0.05$; $3-P_{a}=0.01$.

As can be seen from Figure 2, the vibration amplitude of the droplet surface resonantly increases in approaching the frequency of natural oscillations, and the width of resonance depends on pump amplitude. However, for the practical use of Equation (14), it is sufficient to obtain the value of the vibration amplitude in three limiting cases [36]: (1) $\Omega<\omega$, (2) $\Omega>\omega$, and (3) $\Omega \approx \omega$.

1. For the high frequencies of ultrasonic irradiation, when $\Omega<\omega$, it follows from Equation (15) that

$$
\frac{\delta R_{m}}{R_{0}}=P_{a} \frac{\Omega^{2}}{\omega^{2}}
$$


2. In the limit concerning a low-frequency acoustic signal when the condition $\Omega>>\omega$ is satisfied, the solution to Equation (14) can be represented as

$$
\frac{\delta R_{m}}{R_{0}}=P_{a}
$$

3. In the resonance $\Omega \approx \omega$, the vibration amplitude of the droplet surface has the maximum value

$$
\frac{\delta R_{m}}{R_{0}}=\left\{\frac{P_{a}}{F(\Omega)}\right\}^{1 / 3}, F(\Omega)=\Gamma_{1}-\Gamma_{1} \Gamma_{2}
$$

Thus, substituting the vibration amplitude of the droplet surface for one of the three possible ranges of the frequency of ultrasonic irradiation using Equation (18), (19), or (20) into Equation (14), the dynamics of ultrasonic extraction can be traced by the behavior of a separate element of the breakup of the original solution containing the target component. If the optimal time of ultrasonic treatment $\tau_{0}$ is determined based on the number of extracted particles $\varepsilon N_{0}(0<\varepsilon<1)$, it can be written from Equation (13) that:

$$
\tau_{0}=\frac{\pi R_{0}}{6 \delta R_{m} \omega} \ln \left(\frac{1}{\varepsilon}\right)
$$

It follows from Equation (21) that, specifying the final concentration of particles being extracted that is required at the end of ultrasonic treatment, it is possible to determine the necessary time of acoustic action for the chosen intensity and frequency of ultrasound. It is inversely proportional to the amplitude of acoustic pressure, formally becoming infinite as external action decreases to zero. Such development of events is never implemented in practice, and extraction takes place for a finite time due to diffusion and convection, which were excluded from the present consideration. Equation (21) also indicates a reduction in the treatment time with a decrease in the size of a droplet and an increase in the ultrasound frequency inside the diapason of frequencies $\Omega>{ }^{2} \omega$ used in experiments. However, in the general case, the dependence on the frequency is more complicated since the amplitude $\delta R_{m}$ depends on $\omega$ what corresponds to Equations (18)-(20). The parameter $\varepsilon$ that characterizes the degree of extraction of the target component is under the logarithm sign in (21); therefore, its variation weakly affects the duration of treatment, i.e., extractions by $30 \%$ and $60 \%$ take place with a comparably small difference in time.

The solution of Equation (9) in the form of Equations (18)-(20) is valid over a wide range of the frequencies of stimulating ultrasonic irradiation and its intensities except for the narrow region of parametric resonance when $\omega \approx 2 \Omega$. When the conditions of such a resonance are satisfied, there is an exponentially rapid increase in the amplitude of natural oscillations of the droplet surface even at a very weak intensity of ultrasound. However, for detailed consideration of the specific features of this process, a special separate analysis should be performed.

\section{Results and Discussion}

The dependence of the interphase distribution of sulfosalicylic acid (Chimmed, Moscow, Russia) (the initial concentration is $0.0028 \mathrm{~mol} / \mathrm{L}$ ) in an aqueous two-phase system (polyethylene glycol 1500 (15 wt \%)- $\mathrm{Na}_{2} \mathrm{SO}_{4}(9 \mathrm{wt} \%)$-water) on time with and without ultrasound was experimentally studied. Polyethylene glycol 1500 and sodium sulfate produced by Sigma-Aldrich, Saint Louis, MO, USA. For the preparation of the system, distilled water was used.

An experimental setup is presented in Figure 3. 


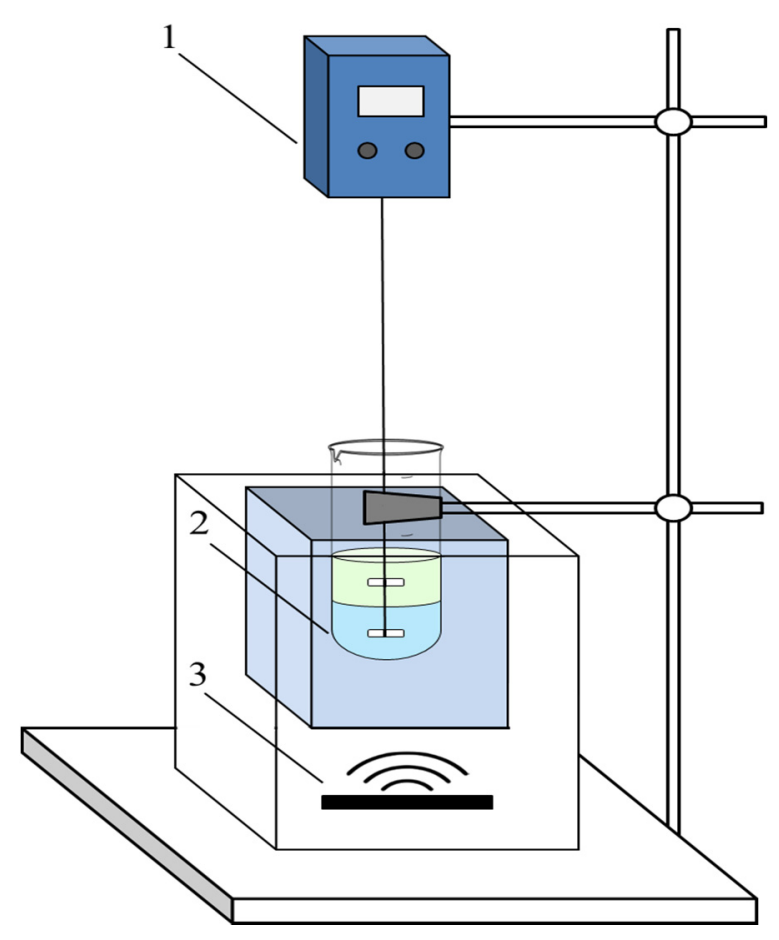

Figure 3. Experimental setup: 1-top-driven stirrer; 2 - extraction system; 3-ultrasonic generator.

Acid extraction experiments were carried out in a glass cylinder (with a constant interfacial area value of $5.31 \mathrm{~cm}^{2}$ ) with mixing of equal volumes $(15 \mathrm{~mL}$ ) of salt and polymer phases. Recoverable acid with a given concentration was prepared in the salt phase. Experiments were performed at a temperature of $25{ }^{\circ} \mathrm{C}$. For providing equable target component distribution into the whole volume, mixing of both phases was carried out with a topdriven stirrer at a speed of $100 \mathrm{rpm}$. An ultrasonic generator (Grad Technology, Moscow, Russia) with a power of $110 \mathrm{~W}$ and a frequency of $35 \mathrm{kHz}$ was used to perform experiments with ultrasound. Quantification of sulfosalicylic acid was carried out spectrophotometrically in the UV region at a wavelength of $230 \mathrm{~nm}$ with Cary 60 spectrophotometer (Agilent Tech., Santa Clara, CA, USA). Acid detection in experiments without ultrasonic treatment was carried out online in a cylinder using a fiber-optic probe with an optical path of $10 \mathrm{~nm}$ every $10 \mathrm{~s}$. In ultrasonic-assisted experiments detection of absorbance was carried out every minute in the initial portion of the dependence until the constant values of absorbance were established, after that measurement frequency reduced to $5 \mathrm{~min}$. Spectrophotometer control and obtained data processing were carried out with Cary WinUV software (Agilent Tech., Santa Clara, CA, USA). All presented experimental data were the result of a series of experiments and processed by the methods of mathematical statistics.

The results of extraction obtained in experiments with and without ultrasound were compared with the calculated data described by Equation (14). Based on this formula for the time-varying density $\delta n(\tau)$ of the substance being extracted from a droplet (or simultaneously from a multitude of droplets), the following equation can be written:

$$
\delta n(\tau) \cong D\left[1-\exp \left\{-6 \delta R_{m} \omega \tau /\left(\pi R_{0}\right)\right\}\right]
$$

Here, $D$ is the maximum density of the substance being extracted that can be recovered by the purification methods used. The theoretical curves that show the function $\delta n(\tau)$ in accordance with Equation (22) in Figure 4 were obtained for different amplitudes of ultrasound and $\alpha=0.075 \mathrm{~kg} / \mathrm{s}^{2}, \omega=35 \mathrm{kHz}, D=8.5$. 


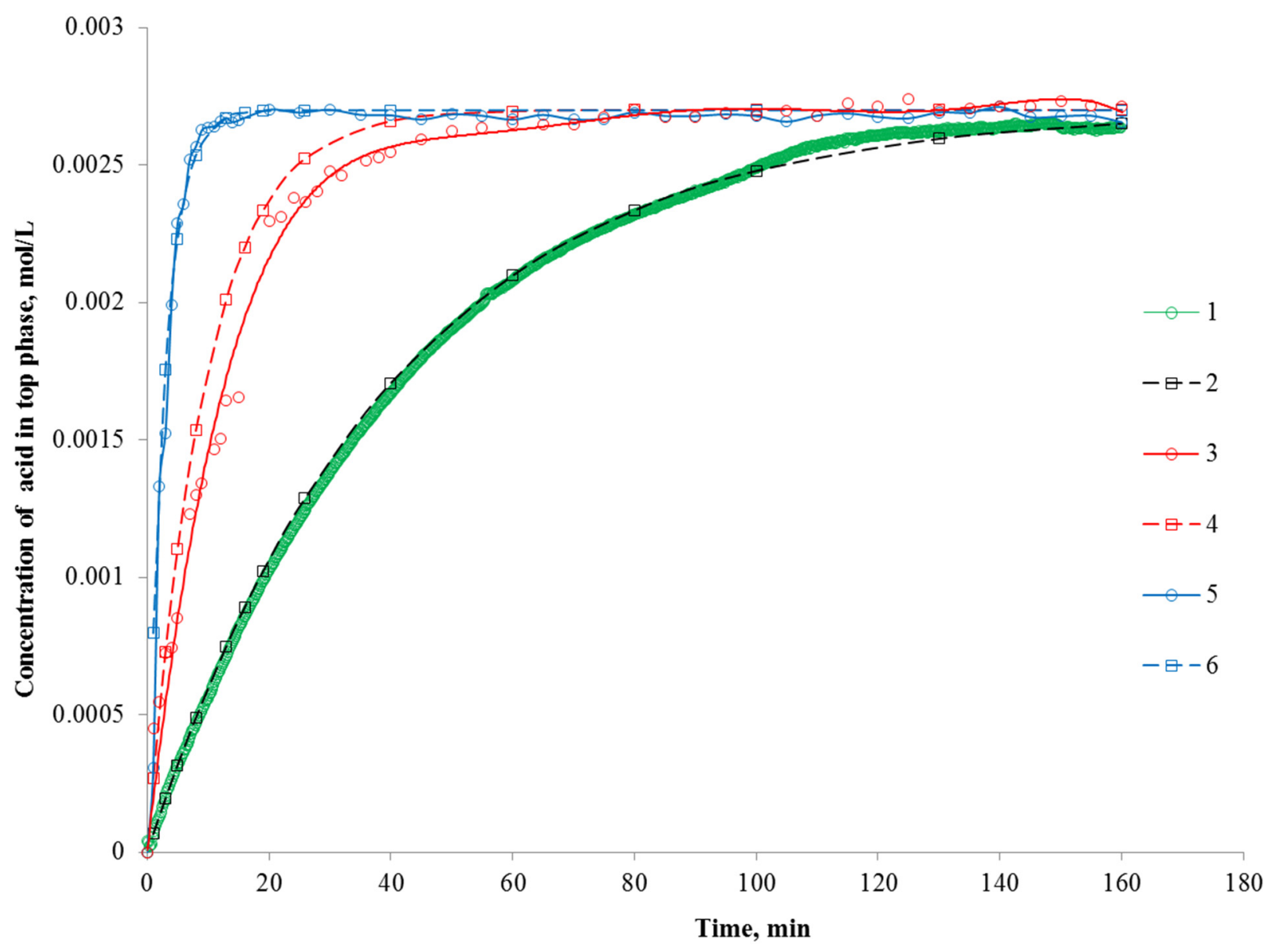

Figure 4. Comparative results of the extraction of sulfosalicylic acid using the PEG-1500- $\mathrm{Na}_{2} \mathrm{SO}_{4}-\mathrm{H}_{2} \mathrm{O}$ system with and without ultrasound and their agreement with theoretical calculations: $1.2-P_{m}=0 ; 3.4-P_{m}=0.6$ bar; $5.6-P_{m}=2.0$ bar (experimental curves—solid line; theoretical curves—dashed line).

The important point is that the functional time dependence of the experimentally obtained quantity $\delta n(\tau)$ is in exact agreement with the analytical description in Equation (22). The almost complete coincidence of these curves at the stage of intensive extraction indicates the adequacy of the used representation of the physics of the processes under discussion to the considered reality. The result of ultrasound in the form of some intensification of the extraction process at the initial stage has a simple physical explanation. Figure 5 shows the state of a drop with an extractable substance, both in the absence of ultrasound and in the presence of variable pressure in the environment. In the case of exposure to ultrasound, the time required to reach the phase boundary (drop border) is less than in the absence of ultrasonic exposure. This is because the drop radius oscillates, and this time is reduced not only by reducing the distance to the phase boundary but also due to the resulting movements inside it. 


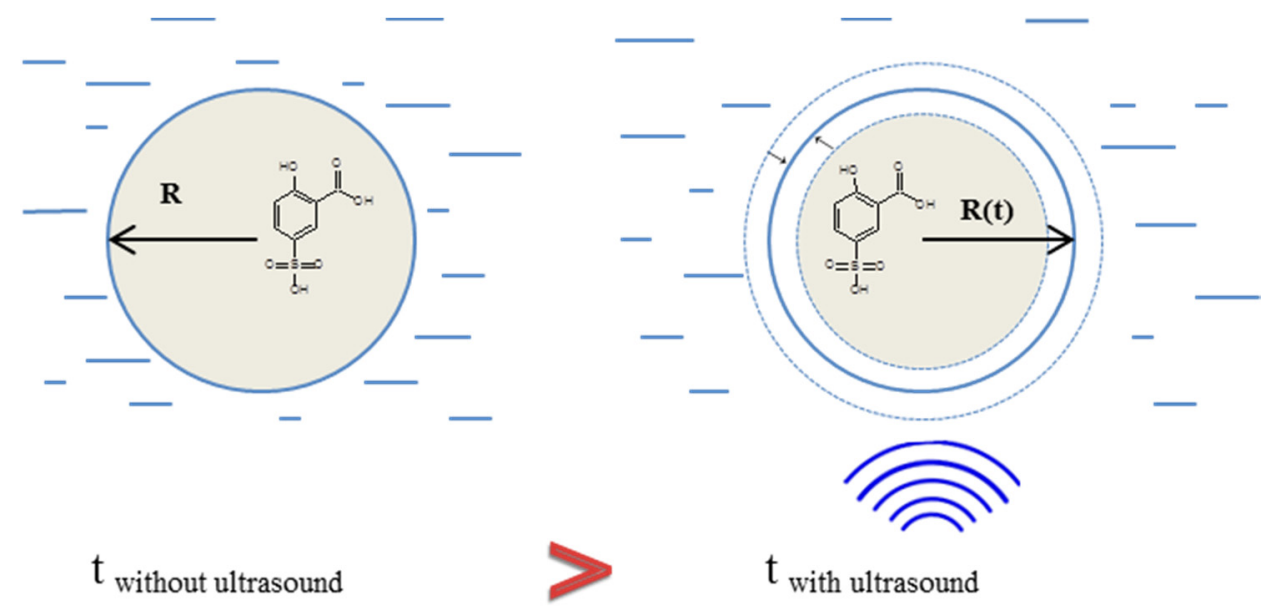

Figure 5. Positions of a drop in the absence of ultrasound and in the presence of variable pressure in the environment.

\section{Conclusions}

The results of this work make it possible to formulate several conclusions that generalize the most important outcomes of the conducted studies:

1. Simple consideration of the behavior of a droplet of the dispersed phase in an immiscible continuous phase under ultrasonic irradiation, even without allowance for its effect on the mechanisms of the recovery of the compound being extracted, when only the variation of conditions on the surface where mass transfer takes place is taken into account, already leads to understanding the existence of the noticeable effect of acoustic radiation on the intensity of extraction. The role of this effect on the intensification of extraction can be considerably increased if conditions are implemented in which ultrasound can directly affect the mechanisms of mass transfer. Therefore, the study of the ability of ultrasound to directly change, under certain conditions, the character of intermolecular interactions between the substance being extracted and the extractant in each mass transfer event is quite important and promising.

2. The performed analysis of the specific features of the effect of ultrasound on a separate droplet shows that changing such ultrasound parameters as frequency and intensity, it is possible to substantially affect extraction processes by controlling their rate. Using the potential of ultrasound to affect the mechanism of recovery of the compound being extracted from a solution, it is possible to determine conditions for the activation of this process and to extract substances that cannot be recovered without acoustic action. However, all of this requires conducting additional studies.

3. The derived relationships for the duration of ultrasonic treatment of an extraction system and the vibration amplitude of the droplet surface can help to considerably increase the efficiency of the use of ultrasound. At the same time, a strongly pronounced resonance character of the dependence of processes associated with acoustic action on its frequency can also be used to develop certain schemes of ultrasonic treatment to obtain characteristics of the extraction process.

4. The revealed good agreement between the results of the experiment and calculated data indicates that the real process of ultrasonic extraction develops in strict accordance with the function that is the result of calculations based on a certain representation of the predominant mechanisms that underlie this process. Therefore, this fact, which proves the validity of applied theoretical premises, can be used decisively for revealing and subsequent practical implementation of other interesting possibilities opened as a result of the targeted use of ultrasound in extraction processes.

Author Contributions: Conceptualization, O.M.G. and A.A.V.; Methodology, O.M.G.; Software, A.A.V.; Validation, A.A.V. and Y.A.Z.; Formal Analysis, Y.A.Z.; Investigation, I.V.Z.; Resources, 
O.M.G. and Y.A.Z.; Data Curation, I.V.Z.; Writing-Original Draft Preparation, O.M.G.; WritingReview \& Editing, Y.A.Z.; Visualization, A.A.V. All authors have read and agreed to the published version of the manuscript.

Funding: This work was supported by IGIC RAS state assignment.

Conflicts of Interest: The authors declare no conflict of interest.

\section{References}

1. Chemat, F.; Rombaut, N.; Sicaire, A.G.; Meullemiestre, A.; Fabiano-Tixier, A.S.; Abert-Vian, M. Ultrasound assisted extraction of food and natural products. Mechanisms, techniques, combinations, protocols and applications. A review. Ultrason. Sonochem. 2017, 34, 540-560. [CrossRef] [PubMed]

2. He, Y.; Zeng, Y.; Chen, K.; Ullah, E.; Li, S.; Zhang, L.; Ren, S. Solvent extraction performance of Sm (III) using a T-junction microreactorwith 2-ethylhexyl phosphonic acid mono-2-ethylhexyl (EHEHPA). Chem. Eng. Process. Process Intensif. 2019, 136, 28-35. [CrossRef]

3. El-Hefny, N.E. Kinetics and mechanism of extraction of $\mathrm{Cu}(\mathrm{II})$ by CYANEX 302 from nitrate medium and oxidative stripping of $\mathrm{Cu}(\mathrm{I})$ using Lewis cell technique. Chem. Eng. Process. Process Intensif. 2010, 49, 84-90. [CrossRef]

4. Zakhodyaeva, Y.A.; Izyumova, K.V.; Solov'eva, M.S.; Voshkin, A.A. Extraction separation of the components of leach liquors of batteries. Theor. Found. Chem. Eng. 2017, 51, 883-887. [CrossRef]

5. Belova, V.V.; Egorova, N.S.; Voshkin, A.A.; Khol'kin, A.I. Extraction of rare earth metals, uranium, and thorium from nitrate solutions by binary extractants. Theor. Found. Chem. Eng. 2005, 49, 545-549. [CrossRef]

6. Belova, V.V.; Voshkin, A.A.; Egorova, N.S.; Khol'kin, A.I. Extraction of rare earth metals from nitrate solutions with a binary extractant based on Cyanex 272. Russ. J. Inorg. Chem. 2010, 55, 629-633. [CrossRef]

7. Palma, M.; Barbero, G.F.; Piñeiro, Z.; Liazid, A.; Barroso, C.G.; Rostagno, M.A.; Prado, J.M.; Meireles, M.A.A. Extraction of natural products: Principles and fundamental aspects. In Natural Product Extraction: Principles and Applications; Rostagno, M.A., Prado, J.M., Eds.; The Royal Society of Chemistry: Cambridge, UK, 2013; pp. 58-88.

8. Bligh, E.G.; Dyer, W.J. A rapid method of total lipid extraction and purification. Can. J. Biochem. Physiol. 1959, 37, 911-917. [CrossRef]

9. Folch, J.; Lees, M.; Stanley, G.H.S. A simple method for the isolation and purification of total lipides from animal tissues. J. Biol. Chem. 1957, 226, 497-509.

10. Zakhodyaeva, Y.A.; Voshkin, A.A.; Belova, V.V.; Khol'kin, A.I. Extraction of monocarboxylic acids with binary extracting agents based on amines and quaternary ammonium bases. Theor. Found. Chem. Eng. 2011, 45, 739-743. [CrossRef]

11. Belova, V.V.; Kulichenkov, S.A.; Voshkin, A.A.; Khol'kin, A.I.; Kuvaeva, Z.I.; Soldatov, V.S. Extraction of mineral acids with methyltrioctylammonium dinonylnaphthalenesulfonate. R.J. Inorg. Chem. 2007, 52, 460-464. [CrossRef]

12. Voshkin, A.A.; Zakhodyaeva, Y.A.; Zinov'eva, I.V.; Shkinev, V.M. Interphase distribution of aromatic acids in the polyethylene glycol-sodium sulfate-water system. Theor. Found. Chem. Eng. 2018, 52, 890-893. [CrossRef]

13. Zinov'eva, I.V.; Zakhodyaeva, Y.A.; Voshkin, A.A. Data on the extraction of benzoic, salicylic and sulfosalicylic acids from dilute solutions using PEG-based aqueous two-phase systems. Data Brief 2020, 28, e105033. [CrossRef]

14. Zinov'eva, I.V.; Zakhodyaeva, Y.A.; Voshkin, A.A. Interphase distribution of caffeine and coumarin in extraction systems with polyethylene glycol and sodium sulfate. Theor. Found. Chem. Eng. 2019, 53, 996-1000. [CrossRef]

15. Zakhodyaeva, Y.A.; Solov'ev, V.O.; Zinov'eva, I.V.; Rudakov, D.G.; Timoshenko, A.V.; Voshkin, A.A. Interphase distribution of thiophene, toluene, and o-xylene in the hexane-polymer-water extraction system. Theor. Found. Chem. Eng. 2019, 53, 550-555. [CrossRef]

16. Mullakaev, M.S.; Vexler, G.B.; Mullakaev, R.M. Sonochemical technology for separating oil sludge and oil-contaminated soil. Pet. Sci. Technol. 2018, 36, 604-608. [CrossRef]

17. Abramov, V.O.; Abramov, O.V.; Sommer, F.; Gradov, O.M.; Smirnov, O.M. Surface hardening of a metals by ultrasonically accelerated small metal balls. J. Eng. Appl. Sci. 1998, 36, 1013-1019. [CrossRef]

18. Newman, A.P.; Lorimer, J.P.; Mason, T.J.; Hunt, K.R. An investigation into the ultrasonic treatment of polluted solids. Ultrason. Sonochem. 1997, 4, 153-156. [CrossRef]

19. Entezari, M.H.; Kruus, P. Effect of frequency on sonochemical reactions. I: Oxidation of iodide. Ultrason. Sonochem. 1994, 1, S75-S79. [CrossRef]

20. Dashamiri, S.; Ghaedi, M.; Dashtian, K.; Rahimi, M.R.; Goudarzi, A.; Jannesar, R. Ultrasonic enhancement of the simultaneous removal of quaternary toxic organic dyes by $\mathrm{CuO}$ nanoparticles loaded on activated carbon: Central composite design, kinetic and isotherm study. Ultrason. Sonochem. 2016, 31, 546-557. [CrossRef]

21. Wen, C.; Zhang, J.; Zhang, H.; Dzah, C.S.; Zandile, M.; Duan, Y.; Luo, X. Advances in ultrasound assisted extraction of bioactive compounds from cash crops-A review. Ultrason. Sonochem. 2018, 48, 538-549. [CrossRef]

22. Zhong, K.; Wang, Q. Optimization of ultrasonic extraction of polysaccharides from dried long an pulp using response surface methodology. Carbohydr. Polym. 2010, 80, 19-25. [CrossRef]

23. Tor, A.; Aydin, M.E.; Ozcan, S. Ultrasonic solvent extraction of organochlorine pesticides from soil. Anal. Chim. Acta 2006, 559, 173-180. [CrossRef] 
24. Diehl, L.O.; Gatiboni, T.L.; Mello, P.A.; Muller, E.I.; Duarte, F.A.; Flores, E.M. Ultrasound-assisted extraction of rare-earth elements from carbonatite rocks. Ultrason. Sonochem. 2018, 40, 24-29. [CrossRef] [PubMed]

25. Makos, P.; Przyjazny, A.; Boczkaj, G. Hydrophobic deep eutectic solvents as "green" extraction media for polycyclic aromatic hydrocarbons in aqueous samples. J. Chromatogr. A 2018, 1570, 28-37. [CrossRef] [PubMed]

26. Saien, J.; Daneshamoz, S. Experimental studies on the effect of ultrasonic waves on single drop liquid-liquid extraction. Ultrason. Sonochem. 2018, 40, 11-16. [CrossRef] [PubMed]

27. Amiri, S.; Shakeri, A.; Sohrabi, M.R.; Khalajzadeh, S.; Ghasemi, E. Optimization of ultrasonic assisted extraction of fatty acids from Aesculus hippocastanum fruit by response surface methodology. Food Chem. 2019, 271, 762-766. [CrossRef]

28. Hu, Y.; Kwan, T.H.; Daoud, W.A.; Lin, C.S.K. Continuous ultrasonic-mediated solvent extraction of lactic acid from fermentation broths. J. Cleaner Prod. 2017, 145, 142-150. [CrossRef]

29. Dordevic, T.; Antov, M. Ultrasound assisted extraction in aqueous two-phase system for the integrated extraction and separation of antioxidants from wheat chaff. Sep. Purif. Technol. 2017, 182, 52-58. [CrossRef]

30. Voshkin, A.A.; Gradov, O.M. Parametric splitting and transfer of liquid cuts for the intensification of mass exchange in a cylindrical volume. Theor. Found. Chem. Eng. 2017, 51, 274-281. [CrossRef]

31. Gradov, O.M.; Voshkin, A.A.; Zakhodyaeva, Y.A. Analysis of the possible applications of the acoustic flow effect for the breakup and transfer of liquid substances in a cylindrical volume. Theor. Found. Chem. Eng. 2017, 51, 876-882. [CrossRef]

32. Gradov, O.M.; Voshkin, A.A.; Zakhodyaeva, Y.A. Estimating the parameters of ultrasonically induced mass transfer and flow of liquids in the pseudomembrane method. Chem. Eng. Process. Process Intensif. 2017, 118, 54-61. [CrossRef]

33. Gradov, O.M.; Voshkin, A.A.; Zakhodyaeva, Y.A. Breakup of immiscible liquids at the interface using high-power acoustic pulses. Chem. Eng. Process. Process Intensif. 2018, 131, 125-130. [CrossRef]

34. Flynn, H.G. Physics of acoustic cavitation in liquids. In Physical Acoustics; Mason, W., Ed.; Academic Press: New York, NY, USA, 1964.

35. Ginkll, R.; Ginell, A.M. Tait coefficients of water in humidity and moisture. In Humidity and Moisture: Fundamentals and Standards; Wexler, A., Wildhack, W.A., Eds.; Reinhold Publishing Corporation: New York, NY, USA, 1965.

36. Gradov, O.M.; Zakhodyaeva, Y.A.; Zinov'eva, I.V.; Voshkin, A.A. Some features of the ultrasonic liquid extraction of metal ions. Molecules 2019, 24, 3549. [CrossRef] 